Voix et Images

\title{
Le Roi jaune ou le rat des livres et la folle du logis
}

\section{Jean-Pierre Vidal}

Volume 4, numéro 3, avril 1979

Louis-Philippe Hébert

URI : https://id.erudit.org/iderudit/200168ar

DOI : https://doi.org/10.7202/200168ar

Aller au sommaire du numéro

Éditeur(s)

Les Presses de l'Université du Québec

ISSN

0318-9201 (imprimé)

1705-933X (numérique)

Découvrir la revue

Citer cet article

Vidal, J.-P. (1979). Le Roi jaune ou le rat des livres et la folle du logis. Voix et Images, 4(3), 395-406. https://doi.org/10.7202/200168ar d'utilisation que vous pouvez consulter en ligne.

https://apropos.erudit.org/fr/usagers/politique-dutilisation/ 


\title{
Le Roi jaune
}

\section{ou le rat des livres et la folle du logis}

\begin{abstract}
Deux piliers, qu'il n'était pas difficile et encore moins possible de prendre pour des baobabs, s'apercevaient dans la vallée, plus grands que deux épingles.
\end{abstract}

(Les Chants de Maldoror; Chant IV, strophe II.)

"Mine is a long and sad tale!" said the Mouse, turning to Alice and sighing.

"It is a long tail, certainly", said Alice...

(Alice in. Wonderland, chap. III;)

Dès la couverture malicieusement composée par Micheline Lanctôt, trois Hébert diversement postés, le dernier en point de fuite chapeauté (cheminée, antique coiffe de médecin molièresque?), le premier jaune et couronné aussi de ce titre : "le roi jaune " qui est le nom par ailleurs d'un autre nègre écrivain d'Amérique (Leroi Jones), trois Hébert, three blind mice (un rat se prépare), sur un échiquier où, au dos de la couverture, une reine fait bouffer sa robe sur sọn corps vautré, trois Hébert disent qu'il y a du jeu, du rouage (jaune) et peut-être ce très fameux "nain jaune", «jeu de carte, comme dit l'autre (Robert), où l'on place les mises sur un tableau dont la case centrale représente un nain vêtu de jaune, qui tient à la main un sept de carreau ${ }^{1}$ "'.

'Portrait de l'artiste en jeune jaune roi nain? Affaire à suivre.

Mais c'est déjà, pages tournées, . "l'histoire qui va suivre " et qui est, paraît-il, a tranchée par le nombril ". Le chapeau de médecin s'impóse à la couverture ${ }^{2}$, c'est donc l'accoucheur du roi ou le roi accoucheur mais jaune résolument toujours. Mythe cliché de l'écriture, écrire c'est pondre (il y aura bien des œufs dans ce texte, mais pas tous dans le même panier, de ces oeufs dont on ne fait pas les omelettes sans quelque peu les casser), raconter c'est accoucher. 
On le voit, la langue d'Hébert et sa fiction débridée roulent dans les marges, en sous-main, bien des formules. Par exemple, «tranché par le nombril " c'est aussi, par manière de clin d'œil, un substitut de «tiré par les cheveux". L'humour d'Hébert : allusif, en coin, comme un ris jaune.

Tirées par les cheveux incontestablement, à la Dodgson, à la Ducasse, à la Roussel, entre autres, les histoires qui vont suivre.

Ah, nos petits journaux jaunes et leur démence fabulatoire!

Mais ici le roi n'est pas nu, il est jaune, de l'or que son titre implique et porte même en son corps signifiant. Et cet univers de légende, de contes à dormir debout (on pense incessamment au comte de Lautréamont et à son "conte somnifère"), comme chez Lautréamont justement, livre ses lois d'emblée, dès l' "adresse au peuple" qui est un avis au lecteur «avant les grandes tourmentes" (Lautréamont proposait aussi, dès la strophe 1 du Chant 1, entre autres métaphores du texte, la tempête).

\section{L'adresse : du roi jaune au rat bleu}

Jeu de couleurs d'abord, successivement: jaune (le roi), bleu ( «le petit animal»), orange (ici sous la forme du fruit ${ }^{3}$ ), bleu («le petit animal», encore), vert ("les pois"), blanc ("un vieillard»), bleu ("un rat»). Encore ne faut-il pas oublier les couleurs implicites: celles, au choix, du système pileux ("cheveux", "poilues", "barbe»), celles, au choix encore, mais des saisons et essences, des arbres ${ }^{4}$ qui sont dans le décor, celles enfin du feu ( «ils ouvriront des cigares. La cathédrale du coin de la rue brûlera»). Spectre mobile dont le Roi jaune sait se faire kaléidoscope.

Jeu de lettres aussi, par exemple le paragramme de «roi jaune»: "trois histoires de jeunes fille, laissent s'approcher de leurs genoux", entre autres dont les plus secrets: «ils nageront " et «oranges "5. Par exemple, encore, cet autre: "assis près d'une gare [...] ils ouvriront des cigares". Mais il faudrait tout citer car le texte d'Hébert se travaille de lui-même, puzzle sans cesse changeant.

Ce jeu de la lettre forme un système d'échos, d'ondes quasi circulaires, du "que» qui ouvre l'adresse à la «queue» qui la clôt, en passant par les couples «poilues-pois ${ }^{6}$ " et “cerf-volant-cerveau ». Sans oublier cet autre cercle, du "roi jaune" du titre au "rat bleu» de la dernière phrase. Tout citer encore, car le texte d'Hébert évoque ici irrésistiblement l'image de ronds dans l'eau.

Ces diverses logiques textuelfes (et d'autres à élucider) se combinent, s'interpénètrent, s'échangent et forment de leur articulation complexe ce tissu mouvant qu'il vaudrait mieux maintenant redéployer selon sa trame.

«Que ceux qui n'ont pour ventre que trois histoires de jeune fille, laissent s'approcher de leurs genoux le petit animal bleu qui répète: «je suis parti »; ventre et histoire, nous l'avons vu dès l'exergue, sont liés en 
un rapport métaphorique ou métonymique, selon la double lecture que le texte propose. Voilà qu'ici un chiasme complique encore le rapport en réécrivant le texte: "ceux qui n'ont pour histoire que trois ventres...". Plus complexe encore, dans l'ironie, le ventre fait écho à un "vendre» implicite : l'écriture est aussi une opération économique. Tout chavire, on le voit. La charge sémantique explose en tous sens.

Mais nous venons ici de croiser, dans ce jeu ventre-histoire, ce ventriloquisme pour faire bref, une logique numérale qui bien sûr, par la multiplication dans un rapport d'engendrement (un ventre, trois histoires) ressortit au motif de l'accouchement? ${ }^{7}$ mais n'en énonce pas moins, par ailleurs, une structure essentielle: le Roi jaune c'est quatre ${ }^{8}$ livres, relativement autonomes, dont chacun comporte trois parties, elles-mêmes formées d'un nombre variable de textes. Cette déclaration du roi ne laisse décidément pas d'être programmatique.

Autre programme qui tient au ventre: la nourriture. On mange ou plutôt on déguste beaucoup chez Hébert. Fruits et légumes surtout y sont pivots essentiels de l'écriture et du rêve (tout particulièrement dans Textes extraits de vanille). Ici, dans cette adresse, l'orange et les pois verts mais il y en aura bien d'autres dans le Roi jaune.

Dans ce contexte de ventre, ce qui se dit «parti" (comme expulsé, né, mais aussi en "trip" - après la tripe - par quelque drogue, au moment où le texte démarre, c'est un "petit animal bleu", produit de rêve (psychédélique ou plus sagement légendaire, comme dans «oiseau bleu», "fleur bleue", etc.) et signe d'écriture ${ }^{9}$, l'imaginaire, l'imagerie d'Hébert ne sont pas seulement ménagers ${ }^{10}$, il joue aussi la ménagerie. Dans le Roi jaune, parmi une pléthore animale (dont de savoureux hybrides imaginaires), outre le rat, le singe est roi. Le singe, autant dire l'anagramme de signe $^{11}$, qui répercute peut-être le portrait de l'artiste en jeune singe de Butor mais qui surtout, avec le rat précisément, appartient à la race élue de ceux que manipulent, mutilent et modifient les laboratoires humains ${ }^{12}$. L'écriture, non plus comme la tauromachie de Leiris, mais comme une vivisection. II faut ici signaler l'importance que revêtent dans l'œuvre d'Hébert les motifs liés de l'expérimentation et de la mutilation (souvent modulée en chute «naturelle» d'organes ou en infirmité : l'hôpital est. un lieu essentiel, la prothèse un objet-signe capital) ainsi que ce qui est peut-être leur résultante: la machine, restriction ou excroissance du corps, torture ou euphorie de l'imaginaire, mais célibataire toujours ${ }^{13}$.

Dans cette adresse du roi, l'animal rejoint la machine, représentée sous une forme rudimentaire par le «cerf-volant " qui justement "représente en un seul dessin le petit animal bleu comme une boite de conserve". Nous abordons ici à un autre centre (ventre) du discours d'Hébert: l'enchâssement ou les jeux topologiques. En effet, la séquence qui fait apparaître le cerf-volant se lit comme suit : “Ceci dit pour n'effrayer que les interlocuteurs valables, assis près d'une gare où les convois d'oranges ne 
tarderont plus, devancés comme ils le sont par quarante majorettes poilues et quarante visages de femmes, le chapeau sur la poitrine, sans oublier par la fenêtre le cerf-volant qui plane dans leur tête et représente en un seul dessin le petit animal bleu comme une boîte de conserve avec les pois verts qui s'en détachent et bouchent les trous du cerveau. "

Le "petit animal bleu" apparu d'abord dans une séquence.de départ ( “je suis parti ») reparaît ici dans une séquence d'arrivée attendue. La gare en découle, comme elle produit "oranges" en paragramme. Mais la gare découle aussi de l'adresse "avant les grandes tourmentes" et de cette formule: "ceci dit pour n'effrayer que les interlocuteurs valables", elle en découle par l'infratextuel «gare!».

Ce n'est pas tant la double ou triple justification de la gare que la stratégie du lieu ainsi mise en œuvre qui doive nous retenir iç. Car la gare est bien ce type de lieu paradoxal de la topologie : elle contient et ne contient pas les interlocuteurs, elle contient et ne contient pas les convois dont l'attente est représentée par ce qui les précède: les majorettes ${ }^{14}$. Quant au cerf-volant, il est à la fois "par la fenêtre" et "dans leur tête". Mais la tête de qui? Et le chapeau de qui? La construction de la phrase est telle que le chapeau et la tête peuvent aussi bien ressortir aux «interlocuteurs " qu'aux “majorettes", elles-mêmes en tête des convois. II n'est même pas dit que chapeau et tête appartiennent à la même instance.

Et les jeux d'espace ne s'arrêtent pas là : le cerf-volant qui est dans la tête et par la fenêtre porte un dessin qui est et n'est pas «le petit animal bleu ", d'abord parce que précisément c'est un dessin et surtout parce que ce dessin admet une comparaison : "une boîte de conserve" (un espace clos) mais "avec les pois verts qui s'en détachent" (un espace ouvert ou excédé). Cela devient vertigineux quand les pois qui sont (et ne sont pas) dans la boîte ${ }^{15}$ qui est (et n'est pas) sur le cerf-volant qui est à la fois dans la tête et par la fenêtre - I'homme qui a vu l'homme qui a vu l'ours quand ces pois, dis-je, finissent par colmater "les trous du cerveau " qui, cela va de soi, est lui-même dans la tête. Mais dạs la tête de qui? Da capo à la coda.

Sans queue ni tête l'histoire parce que la fiction est le produit d'un déboîtement perpétuel de ses árticulations signifiantes, articulations dont le jeu finit par capturer ce qui les déclenche. Les récits d'Hébert ne sont pas (ou très rarement) récits de quelque chose mais un tentacule d'écriture qui se déploie et pousse à la recherche d'une proie indéfinie, une proie qui est à la fois sa proprè matière et la projection d'une ombre d'histoire $^{16}$ : une fiction de légende et de rêve. Kaléidoscope, puzżle, mécanique ondulatoire, topologie: c'est l'univers des anamorphoses que l'écriture en son parcours fait surgir.

Mais qu'est-ce qui fait donc tenir ensemble toutes ces métamorphoses? Quelle syntaxe, quel «liant " en assure la crédibilité ou même, simplement, la lisibilité? 
C'est justement, me semble-t-il, une syntaxe familière ${ }^{17}$ et, plus encore, un petit air de famille avec non seulement le discours des proverbes et des fables, mais tel ton sentencieux de. l'expérience commune, dès lors qu'elle se soucie de se généraliser.

Et ici encore Lautréamont (ou Lewis Carroll) n'est pas loin. Comme chez Lautréamont, la rigueur de la relation, la logique imperturbable (je dirais même pataphysique) de son articulation accréditent les rencontres les plus folles; les rapprochements les plus saugrenus. C'est, par exemple, dans cette adresse; le "ceux qui n'ont pour ventre" qui fait passer le "trois histoires de jeunes filles" et tout ce qui s'ensuit. Le déictique "ceux", croisant le lot universel du "ventre", subverti d'un espace métaphorique («pour ventre») et d'une restriction ("pour ventre que »), assoit ún discours de vérité, légèrement tremblée de la subversion que je viens d'énoncer, un discours de vérité qui fait de tout ce qui suit «parole d'évangile», d'évangile textuel évidemment, c'est-à-ḍire de contre-évangile. Unè sorte de syllogisme ironique et fou sous-tend cette phrase : tous les hommes ont un ventre, certains en ont plusieurs, quant à ceux qui n'en ont que trois... Chacun des termes, on le voit, s'aśsure du précédent, explicite oú implicite. Et ce qui passe, en contrebande, ce n'est pas tant la possible multiplicité des ventres, que leur capacité d'être histoire!

La dimension proverbiale ou plus généralement sentencieuse est èssentielle à la pratique scripturale d'Hébert. Une sorte d'appel à tous, de prise à témoin ${ }^{18}$ qui joue de l'œcuménisme de toutes les morales de fables, mais qui fait passer en lieu. et place du discours de vertu celui du rêve, infiniment pervers.

Si cette analyse n'a guère jusqu'à présent dépassé la page initiale tout en pouvant prétendre avoir parlé du texte tout entier, c'est à une autre caractéristique du' travail d'Hébert qu'elle le doit. Dans le Roi jaune et dans l'ensemble de l'œuvre, tout texte est à la fois irréductible et repris, perpétué pár d'autres, contemporains ou pluśs récents même. Cette œuvre se lit éllemême, car elle est toute entière sous le signe de la variation, ou du "plagiat " de Lautréamont ${ }^{19}$. D'un texte mille peuvent se faire, car chacun est comme, un organisme en suspension, dont l'équilibre est instable, provisoire et peut se défaire pour se reformer autre, ailleurs ou dans son propre espace par le contre-coup d'un autre texte nouvellement apparu. D'où la surprise et le plaisị de ceux qui, comme moi, ont abordé Hébert par ses textes récents et retrouvent dans le Roi jaune bien des choses qu'ils croyaient faịre partie d'autres livres et qui certes y sont mais sans manquer pour autant d'être déjà dans le Roi jaune mais différentes, déplacées, autres alors, changées dès lors, tremblées en quelque sorte.

Le Roi jaune est comme une coupe de l'œuvre entière d'Hébert: c'est à partir de lui que ce qui était poème devient texte bref et c'est en lui déjà que se décèlent les deux “moments" de la pratique hébertienne du texte bref : tantôt poème en prose en ce sens qu'aucune fiction unifiée 
ne parvient à y «prendre», chaque fiction circonscrite à la phrase ou au syntagme qui la porte, tantôt court récit où le travail du texte, sans cesser d'être un seul instant essentiel, concourt à l'élaboration d'une fiction homogène, d'une nouvelle avec chute bien souvent, qui se peut raconter et fait surgir alors les pairs d'Hébert du côté des maîtres américains, Poe, Bierce, Lovecraft ou des Cortazar, Borges, Buzzati. II semble que, dans les œuvres récentes, et notamment les inédits, cette dernière tendance prenne légèrement le dessus sur l'autre.

Particulièrement exemplaire de cette tendance, dès le Roi jaune, le texte intitulé "petit déjeuner sur l'herbe" (p. 240).

Mais aussi le livre premier tout entier où l'on pense souvent à Kafka (celui de la Métamorphose, de la Colonie pénitentiaire, du terrier et du Château, plutôt que celui du Procès ou du moins de l'interprétation massivement symbolique qu'on en donne généralement).

Ce livre premier que nous abordons maintenant de façon résolument plus panoramique.

\section{Le livre premier : l'univers d'Antonio, rat, fils et poète}

La première partie de ce livre premier ${ }^{20}$ s'intitule : “Comment Antonio perdit la raison». Toute entière placée sous le signe du cercle, la roue, les rouages en sont les figures essentielles.

Fiction circulaire ou, pour reprendre l'image de l'adresse, qui se mord la queue, Antonio arrivant au début dans sa demeure, en chaise roulante, la fin le voyant aux prises avec "une chaise roulante où une couverture de laine épaisse attend Antonio " (p. 36).

Cette circularité temporelle, ne serait-ce que de la dimension temporelle du texte, se double d'une circularité spatiale dont la roue est l'emblême. En fait, il faudrait plutôt parler de gyroscope ou de toupie, le mouvement horizontal s'articulant à un mouvement vertical. Ainsi, dès le premier texte. Antonio arrive en chaise roulante, mais par ascenseur (p. 13). Ainsi, la roue, qui au logis où il est roi, le fera rat de laboratoire: “roue à échelle» formée de «deux cerceaux de fer massif réunis l'un à l'autre par les extrémités d'un barreau qui se multiplie à intervalle régulier", duplication des roues de la chaise et roue d'Ézéchiel: "cette circonférence qui pouvait l'inclure tout entier en elle-même, semblait suspendue dans l'air par sa propre force centrifuge" (p. 16). Roue qui permet à Antonio, nouvel «Achille immobile à grands pas (Paul Valéry), de jouir du paradoxe de Zénon le cruel: «... plus elle roulait et plus elle permettait de goûter au double plaisir d'être en même temps au sommet et à la base de la chambre qui s'élevait et redescendait sous ses yeux; toujours en mouvement, il poursuivait son propre corps qui se rapprochait à chaque bond pour le fuir du même coup... » (p. 23). 
La roue d'Antonio n'est en fait que le rouage supérieur d'une gigantesque machine : l'édifice dont il occupe une chambre au "trente-deuxième étage de la quatorzième section ${ }^{21}$ ". L'étage inférieur de cette "ratopolis" évacue à intervalles réguliers des vieilles ${ }^{22}$ qui viennent mourir dehors, un enfant satellite circulant autour d'elles, les "cernant" et semblant de sa course complexe édicter, par quelque mystérieuse influence, leur sort. La marée des vieilles et l'astre-poupon qui l'accompagne ou la provoque entretient avec Antonio des rapports sur le mode de l'observation puis sur celui de la reconnaissance-nomination, dans un curieux passage où l'enfant semble assimilé à Antonio, sous la forme de son inverse nominal: "Déjà un enfant rampait autour d'elles, et le cri vibra longtemps entre les murs lorsqu'elles, lui arrêté vis-à-vis et les fixant tour à tour, le reconnurent: oinotna!" C'est l'échange des regards entre les vieilles et l'enfant qui, dans l'ambiguïté du déictique «le», fait surgir oinotna comme une matérialisation du regard échangé d'Antonio. Un autre rapport unit les vieilles à Antonio : ce sont les mêmes porteurs qui l'avaient hissé en ascenseur sur sa chaise roulante jusqu'à sa chambre qui descendent les mortes en chaise roulante et par un escalier mobile jusqu'aux sous-sols. On voit donc clairement qu'à plusieurs niveaux Antonio et les vieilles forment l'engrenage d'une chaîne mobile qui dispose sur son parcours des figures de la génération. C'est ainsi, de quelque façon, le temps qui s'énonce aussi dans cet espace à l'animation perpétuelle.

Les chambres elles-mêmes tournoient (et dans un rapport à la lumière organisé de telle sorte que, bien qu'horizontal le déroulement, c'est le cinéma qui semble s'en évoquer) et il arrive même à l'immeuble entier de descendre d'un étage. Antonio retrouvant un beau matin sa chambre située au trente et unième étage (p. 28).

Le principe moteur de cette machine immobilière, c'est le nom d'Antonio, son nom inversé, répercuté du cri des vieilles (p. 29) à tous les murs de l'édifice et même à la fin «dans tout le pays" (p. 36).

Il s'agit donc d'une machine qui, par son côté optique (projection du nom), évoque celle de l'Invention de Morel de Bioy Casares et, par son aspect de multiplication du graphe nominal "dans une langue étrangère", reprend la constellation mallarméenne du Coup de dé.

Qu'est-elle donc, en vérité, cette énigmatique machine sinon justement, comme les précédentes, une allégorie de la production signifiante ${ }^{23}$, que fait ce rat Antonio sinon l'inversion de son nom Oinotna, de l'art (á partir du rat dont c'est l'anagramme parfait) ${ }^{24}$ ? Sisyphe et Osiris, Antonio-Oinotna perpétuellement s'éparpille, se dissémine, or et excrément ${ }^{25}$, en s'épelant. “A letter, a litter » disait Joyce. Hébert semble ajouter, sur le mode animal : un signe un singe, de l'art un rat. Ce rongeur serait-il frère de la taupe de Marx ${ }^{26}$ ? Suis-je clair?

Mais Antonio n'est pas que rat et écrivain, pas seulement prisonnier et principe moteur, rouage et impulsion, il est aussi simili-fils et pseudofrère. En ligne courbe, par la Reine. 


\section{Une généalogie chavirée: du rat à la reine}

L'organisation sociale, et notamment familiale, que le pays d'écriture du Roi jaune admet en ses fables, ne laisse pas, elle aussi, d'être floue et fluctuante, au gré de la plume.

Pourtant il est possible d'arrêter cette fluctuation et d'y discerner une véritable stratégie. C'est ainsi qu'Antonio apparaît dès le début comme un fils héritier: sa chambre, ce sont ses parents qui la lui ont léguée (p. 14), elle lui appartient de "droit familial" (p. 22). Sa chambre, donc sa fonction, car, si Antonio écrivait déjà avant que d'y entrer (cf. la séquence, déjà citée, dú nom-pourboire), c'est la place qu'il va occuper dans la machine qui transforme ce qui était peut-être une simple vocation (ou un attribut du corps) en fonction et fonction sociale.

Des parents effacés président à son travail-plaisir. Mais cette activité justement le met en rapport, lui le fils sans parents visibles, avec des mères sans enfants avérés: les vieilles avec, au bord de la mort, "ces gestes tranquilles de mères qui ont tout leur temps" (p. 20). Leurs gestes de mères sont dirigés vers l'enfant qui les flaire, les fuit et les dénonce. Or cet enfant, par sa marche à quatre pattes et le trajet cyclique qu'il effectue, est un double d'Antonio, le rat dans sa roue. Et; comme on l'a vu, c'est lui qu'elles nommeront, à travers l'enfant, dans un cri de reconnaissance qui prend la forme de son nom inversé (p. 29).

Puis vient la reine: animée d'une mécanique complexe, "poilue comme un chat" puis couverte de félins (p. 35), avec chevaux puis, "ses chevaux exilés", en chaise roulante elle aussi et vieille irrémédiablement. Ici tout converge: "la main dans les cheveux" qui, partie de l'adresse, ordonnait le geste des vieilles, se transcrit en "chat", "cheval", "chaise" et préfigure les rapports qui dans les deuxième et troisième parties uniront la reine à Antonio: rapports de poil ou plus généralement de fil (tiré par les : cheveux, encore) mais rapports dans tous les cas fondés sur une suite chuintée: cheveux $\rightarrow$ chapeau $\rightarrow$ mouche $\rightarrow$ chameau, etc. Et rapports qui iront, par anamorphose, jusqu'à l'assimilation pure et simple: "quand on est une Reine [...] et qu'on s'appelle Antonio" (p. 48), puis Antonio reprenant à son compte le geste de la main dans les cheveux, Antonio qui «cherch[e] à la peigner» (p. 58), Antonio précédemment devienu le «rat bleu » de l'adresse par son “poil ras bleu » (p. 54).

Qu'est-ce à dire finalement? Antonio et la Reine forment couple, ou plutôt ils sont les deux pôles d'une organisation signifiante, une organisation qui admettra plus tard le Roi jaune puis un «je ", comme Antonio, puis la reine, s'effaceront, que l'on passera du "poil " à la poêle et à l'œuf, que la métaphore de l'engendrement signifiant se transcrira en d'autres figures.

Ces deux pôles, quelles qu'en soient les apparences ponctuelles, me semblent, dans leur complémentarité, leur dialectique même, renvoyer 
aux deux instances, aux deux temps confondus qui ordonnent la pratique scripturale d'Hébert: le jeu de la lettre et son fil dévidé et le jeu de l'imaginaire ou du rêve qui en provient et l'impulse.

La "reine des facultés", la “folle du logis" ne va pas sans quelque travail de rongeur-fouisseur qui, quant à lui, sans elle, ne serait que mécanique taupe. La reine poilue comme un chat trouve son rat ( «à bon chat, bon rat»), une fourrure les rapproche, un œuf les contiendra. Écrire, pour Hébert, c'est peut-être jouer la coquille pour voir ce qui va sortir dé ses éclats.

Le Roi jaune, c'est aussi l'œuf' ${ }^{27}$ de Colomb. Dans sa mire, une Amérique. En français dans le texte.

Jean-Pierre Vidal, Université du Québec à Chicoutimi.

1. J'ajouterai que ce nain, de par son accoutrement, est un bouffon, de l'espèce communément appelée "fou du roi" et que le nain jaune est de ces jeux enfantins et pervers où pour gagner (les mises) il faut perdre (ses cartes). Rien d'étonnant qu'une revue littéraire du XIXe siècle s'en soit trouvé titre congru.

2. Avant de devenir de "cône de papier imprimé qu'il fixe, suprême couronne, sur sa tête» (p. 39). Micheline Lanctôt travaille fort intelligemment sur les postures mythiques de l'écrivain.

3. L'orange est un mot clé de la pratique scripturale d'Hébert. J'y reviendrai.

4. C'est parce que système pileux et végétation s'accordent métaphoriquement dans ce texte que le singe trouvera naturellement place dans les cheveux de la reine (cf. p. 42) ou d'autres personnages (par exemple Antonio, p. 45). Mieux, la reine, à sa première apparition "poilue comme un chat " et d'ailleurs poilue avec constance tout au long du texte, pourra se lire, par croisement avec le singe, poilue comme le dit le cliché: "comme un singe". Chez Hébert, comme chez Lautréamont, le cliché travaille le texte, et de la même façon étrange que chez Lautréamont, le cliché est souvent l'aboutissement, secret ou explicite, du paradoxe scriptural.

5. Ce dernier, outre qu'il répercute le roi aussi, à un autre niveau du signifiant, par la couleur, forme avec "les grandes tourmentes" l'axe : «orage-orange" dont maint texte d'Hébert alors et maintenant encore s'organise. Et ces oranges élucident, par leurs rapports à lui, "le roi jaune» comme ce qui les fait mûrir : le soleil. Réaction en chaîne qu'expérimente toute lecture d'Hébert: le roi jaune c'est le roi-soleil, Louis le quatorzième (jouons du biographique: Louis-Philippe est un nom de roi) ou même, pourquoi pas?, le «Sun King " des Beatles (dans Abbey road).

6. D'ailleurs, toute une séquence, et que la typographie fait quasi verticale, s'en ordonne : "plus, poilues, poitrines, plane, petit, pois ".

7. Le texte accouché produira, dans ce même premier livre du Roi jaune, le texte de "l'accouchement de la Reine-Mère" (p. 67), Reine plus tard verte, comme le Roi est jaune et le rat, leur croisement en quelque sorte, bleu. On n'oublie pas si vite les lois de la palette, fût-on au cceur du palais, et dans des jeux de langue. 
8. Dans cette page initiale, la reprise de l'histoire féminine ("trois histoires de jeune fille") sera marquée par aquarante majorettes poilues, et quarante visages de femmen. Le Roi jaune joue du trois par quatre. Et il ne se lasse pas d'hominiser les dames, de féminiser les messieurs et d'animaliser tout le monde. Le travesti est une hantise du texte moderne, sinon de ses producteurs (n'allons pas soupçonner les mœurs - qui en ont vu d'autres, il est vrai de nos littérateurs), car il est la plus parfaite métaphore de la production signifiante qui se puisse concevoir. $\mathrm{Cf}$. au Québec, les textes de Nicole Brossard et ceux de Roger des Roches, entre autres French kiss et Reliefs de l'arsenal.

9. On voudra bien se reporter à mon deuxième exergue où l'histoire de la souris est prise, par Alice, pour sa queue. C'est une des nombreuses traces d'Alice in Wonderland dans l'intertexte du Roi jaune. J'en signalerai d'autres. Pour le moment, remarquons qu'ici l'animal dont le départ coïncide, pour la lecture du moins, avec celui du texte, deviendra le rat qui, lorsque "les interlocuteurs valables" auront "compris", "ne courra plus après sa queue dans les rues pleines de plumes". Manifestement le rat est une métaphore de la fiction circulaire que porte au moins tout le premier livre. D'ailleurs, dans ce premier livre, nous verrons bientôt surgir Antonio, rat et écrivain, entre autres rôles ou masques. Alice donc, et qui croise le lieu commun de l'histoire "sans queue ni tête", du roi de l'en-tête à la queue du rat de fin de page. Petit clin d'œil à Freud dans toute cette panoplie royale et ratière.

10. Le ménager et son temple: la cuisine, comme réservoir et aboutissement du travail d'écriture, du travail du rêve, voilà qui relève d'une certaine malice quand on connaît la place qu'il occupe dans l'édition québécoise... et la culture plus largement occidentale.

11. Le singe est ainsi le double du rat, ce que confirmeront bien des traits de fiction, notamment le fait qu'ils tiennent alternativement l'un et l'autre rang et rôle de "fils bien aimé" et de "chapelier favori" de la Reine. C'est sous le même signe d'une totémisation animale de l'écriture et de l'écrivain que la première apparition de l' "Auteur" (livre troisième, page 225: "L'Auteur évoque la Reine-Mère en termes courtois i) fera venir au texte, quelques lignes plus bas, "une énorme autruche", par le même jeu signifiant que celui qui fait de "roi " «rat».

12. Cf. l'entrevue Morency-Hébert: “Une littérature de laboratoire, bien sûr. Un rat blanc trop bavard distrait le vivisecteur. Suis-je clair?"

13. Cf. à ce propos, ici-même, ci-après : «Une machination du texte " de Ghislain Bourque.

14. Dans le contexte américain qui est le nôtre, ces majorettes précédant des convois d'oranges ne laissent pas d'évoquer peut-être quelque "orange bowl" - dénonçons la lecture et son conditionnement culturel et biographique : j'écris ceci le jour du "Super bowl» - comme les cigares que I'on ouvre plus bas évoquent le rituel américain de la naissance. Ce qui est sûr, du moins, c'est que le cortège des majorettes annonce celui de la Reine (p. 30-31).

15. Ils sont, de toute façon, dans son corps signifiant: boîte < pois, étant donné par ailleurs la proximité phonique $d u$ «b» et $d u$ “p». Cela va même jusqu'au paragramme: «pois verts" $\rightarrow$ aboitte de conserve". A la page 60 , nous retrouverons "boîte» et «poils" à quelques lignes d'intervalle. Une sorte de loi harmonique préside aussi à l'engendrement du texte.

16. C'est une métaphore de ce processus de production signifiante que me semblent représenter : le cinéma, omniprésent chez Hébert (et pas seulement par goût biographique) et ici, dans le Roi jaune, l'écriture d'Antonio: écriture où l'on noircit du noir - reprise modifiée peut-être du noir sur blanc et du blanc sur blanc de Mallarmé : Mallarmé écrit cygne quand Hébert 
trace singe - et où le grattage ainsi produit se lit au verso et à l'envers (“Oinotna" pour "Antonio»), trace du nom qui exige un miroir à la fois traversé et réfléchissant. Écriture sur le mode du négatif et de la surcharge, palindrome et palimpseste à la fois.

17. Mis à part Récits des temps ordinaires et quelques subtiles perversions ça et là, dans les autres textes, Hébert ne détruit pas la phrase canonique. La subversion qu'il fait subir et au texte et au discours se marque ailleurs.

18. Qu'on pourrait chiffrer d'une formule telle: «quiconque a eu l'expérience de... comprendra ce qui suit». Or l'astuce est que, par définition, ce "quiconque» se traduit en fait par “personne» mais il suffit de l'appel et du temps (le passé de l'expérience) qu'il implique pour que toute folie soit avérée, attestée, non par l'impossible confirmation des faits mais par un discours.

19. Le "plagiat" de Lautréamont ne saurait être circonscrit, comme on l'a fait généralement, aux cuures des autres, mais doit s'entendre étendu à la réécriture perpétuelle de son propre texte. Faute de quoi, Ducasse n'est qu'un potache... ou un surréaliste sommaire, ce qui est sensiblement la même chose.

20. C'est dans ce livre surtout que vont se trouver le plus de traces d'Alice in Wonderland: - ses figures animales: souris, chat, lapin etc. - la reine elle-même, non pas, comme dans Alice, reine de cœur sortie d'un jeu de cartes, mais plutôt reine d'échecs à laquelle il arrive de jouer aux cartes (“les passe-temps de la famille», p. 48); - la question de la taille d'un personnage par rapport aux portes qui lui sont offertes: Alice, on s'en souviendra, selon le liquide qu'elle boit, devient trop grande ou trop petite pour la porte mystérieuse. Dans le Roi jaune, Antonio est, lui, parfaitement adapté à une porte plus petite que les autres et la reine (p. 67-69) tente, en se gonflant, d'atteindre certaine porte. Les serrures aussi feront partie d'un jeu qui ressortit à la logique du rêve mais se souvient aussi de l'utilisation qu'en fait le texte de Lewis Carroll; - enfin, la folie d'Antonio, conjuguée au titre de "chapelier" que lui donne la reine, n'est pas sans rappeler le chapitre VII d'Alice ("A mad tea-party") où Carroll prend au pied de la lettre des expressions telles que "mad as a March hare " et "mad as a hatter" pour en faire surgir des personnages: le lièvre de Mars et le chapelier. La folie d'Antonio semble elle aussi le résultat d'une formule qui serait, par exemple, l'expression "perdre la boule", étant donné l'abondance, et pas seulement dans le livre premier, des billes et autres balles que le texte lance perpétuellement. Par ailleurs, cette folie pourrait aussi représenter le «nain jaune" qui est, je l'ai dit, un fou du roi (que le recours au jeu d'échecs peut aussi faire apparaître et même par le biais de son équivalent anglais : le "bishop" qui, mitré, reprendrait le "chapelier"). L'importance, au moins dans le livre premier, des espaces cloisonnés (chambres, immeubles, boîtes) peut évoquer, entre autres (dont le jeu d'échecs) ce jeu où les mises se déposent dans de petits casiers, le casier du nain jaune se plaçant entre celui du roi et celui de la reine: on se reportera à la page 39 où Antonio est présenté avec "le singe et la mère, à sa gauche et à sa droite». Point n'est besoin d'évoquer l'archétype freudien et les lectures qu'il a pu produire (par exemple la désopilante et désolante interprétation par Marie Bonaparte du cdouble assassinat dans la rue Morgue" de Poe) pour faire du singe ici le roi-père. Ce singe qui, par ailleurs, pourra se lire aussi infratextuellement «nain dans les cheveux" puisqu'il prend la place de la "main dans les cheveux" de l'adresse.

21. Chiffre à la Robbe-Grillet: la numérotation et son implicite réalisme de repère se résume en fait à une loi séquentielle dérisoire: $32-14 \rightarrow 1234$, dans le désordre. Subversion du code par son traitement à plat.

22. Il s'agit d'une organisation inverse de celle de la machine célibataire canonique (du type “Mariée mise à nu par ses célibataires, même») puisqu'ici le principe féminin occupe le bas de la machine. 
23. Cf. Entrevue Morency-Hébert: « Je conçois mes livres comme une sorte de quincaillerie pleine de machines/outils, de petits textes/systemes dont on peut se servir à son gré.» Est-il nécessaire d'ajouter que l'interprétation ici proposée de la machine du Roi jaune n'en épuise ni le sens ni le fonctionnement... ni l'usage! Chez Hébert, contrairement à ce qui se passe dans certain texte de Ricardou (les lieux-dits, nommément), la fiction ne se réduit jamais à une métaphorisation du théorique.

24. L'étincelle finale que ma nuit tâtonnante désespérément cherchait, je la dois à Ghislain Bourque auquel soient rendus ici grâces et ce qui à César...

25. Passage significatif, à cet égard: "ll glissa ensuite une main droite dans la poche interne de son cocon pour en sortir du bout des doigts deux boulettes de papier; elles tombèrent en pourboire et s'ouvrirent au contact avec le sol : à l'intérieur, c'est-à-dire écrit sur le côté noir de la feuille froissée, son nom Antonio..." (p. 13).

26. Cf. Entrevue Morency-Hébert: “En ce sens, le geste de tout créateur est politique: en produisant une fiction, il affirme que tout n'est que fiction. En produisant un "système textuel ", il dénonce tous les systèmes."

27. Ai-je vraiment oublié de reparler, comme je me le proposais, de l'orange? Suis-je clair? Allusif, trop? ou prometteur, encore? 